\title{
HOSPITAL-ACQUIRED PNEUMONIA IN ICU PATIENTS
}

\author{
Radovan Uvizla ${ }^{*}$, Vojtěch Hanulik ${ }^{\mathrm{b}}$, Vendula Husickova ${ }^{\mathrm{b}}$, Miroslava Htoutou Sedlakova $^{\mathrm{b}}$, \\ Milan Adamus ${ }^{\mathrm{a}}$, Milan Kolar ${ }^{\mathrm{b}}$
}

\begin{abstract}
a Department of Anesthesiology and Intensive Care Medicine, University Hospital Olomouc and Faculty of Medicine and Dentistry, Palacky University Olomouc, Czech Republic

${ }^{b}$ Department of Microbiology, Faculty of Medicine and Dentistry, Palacky University Olomouc and University Hospital Olomouc
\end{abstract}

E-mail: radovan.uvizl@seznam.cz

Received: July 15, 2011; Accepted with revision: November 24, 2011; Available online: December 19, 2011

Keywords: Hospital-acquired pneumonia/Bacterial pathogens/Resistance/Mortality

Background. This prospective study aimed at assessing the effect of initial antibiotic therapy on the mortality of patients with hospital-acquired pneumonia (HAP) by analyzing bacterial pathogens and their resistance to antimicrobial agents.

Methods. Included were patients hospitalized in the Department of Anesthesiology and Intensive Care Medicine, Faculty of Medicine and Dentistry, Palacký University Olomouc and University Hospital Olomouc in 2009 who developed HAP. Bacterial pathogens and their resistance to antibiotics were identified using standard microbiological methods. The patient's mortality with respect to their initial antibiotic therapy was statistically analyzed.

Results. The group comprised 51 patients with HAP. Early-onset HAP was identified in 7 (14\%) patients and late-onset HAP in 44 (86\%) patients. The most frequent bacterial pathogens were strains of Klebsiella pneumoniae, Pseudomonas aeruginosa, Burkholderia cepacia complex and Escherichia coli, together accounting for $72 \%$. Eighteen patients died directly due to HAP, an overall mortality rate of $35 \%$. If initial therapy effective against the bacterial pathogen was selected, 21 patients survived and 9 died. If the bacterial pathogens were resistant to the selected initial antibiotic therapy, 9 patients died and 12 survived.

Conclusions. The mortality rates were $30 \%$ and $43 \%$ for adequate and inadequate antibiotic therapy, respectively. Given the small group of patients, the difference has low statistical significance. However, it does document the clinical impact of bacterial resistance on the survival or death of patients with HAP.

\section{INTRODUCTION}

Hospital-acquired pneumonia is one of the most frequent and most severe medical complications in patients hospitalized in intensive care units. It develops mainly in association with invasive airway management and mechanical ventilation. It is an important factor affecting health care economy and often related to bacterial resistance. Understanding the etiology of pathogens and their potential resistance to antimicrobial agents has a major impact on both patients' morbidity and mortality and the economic aspect of their treatment ${ }^{1}$.

Pneumonia is diagnosed by assessing clinical signs and detecting etiologic agents. Clinical signs are assessed especially by finding fresh or progressive infiltrates on chest radiographs, together with at least two other signs of respiratory tract infection. From the epidemiological point of view, pneumonia may be either community-acquired (CAP) or hospital-acquired (HAP). CAP is defined by developing in the community outside health care facilities and, if the patient is hospitalized, clinical manifestations within $48 \mathrm{~h}$ of hospital admission. HAP may be characterized as pneumonia developing $48 \mathrm{~h}$ or more after admission to a health care facility or within 14 days after discharge. More precise classification according to the time of the onset of clinical signs distinguishes early-onset (from 2 to 4 days after admission) from late-onset (from day 5 after admission) HAP.

The most common pathogens causing CAP are Streptococcus pneumoniae, Chlamydophila pneumoniae and Mycoplasma pneumoniae ${ }^{2}$. HAP is mostly caused by endogenous bacterial strains, originating from primary microflora or secondary colonizing bacteria with a higher level of resistance in the early- and late-onset forms, respectively ${ }^{1,3}$. This knowledge is used to select the proper therapy since the proportion of individual pathogens determines the strategy of initial antibiotic treatment. Adequate antibiotic therapy is an important factor influencing the effectiveness of therapy and, of course, the patient's survival ${ }^{4,5}$.

The prospective study aimed at identifying etiologic agents of pulmonary inflammation in patients with the most severe forms of respiratory insufficiency requiring mechanical ventilation. The objectives were to describe the prevalence of respiratory tract pathogens, to determine their susceptibility to a range of tested antibiotic agents, and to acquire information on the proportion of resistant strains in the population of these pathogens. The impact of adequate antibiotic therapy was assessed, with respect to patient mortality and morbidity rates. 


\section{MATERIAL AND METHODS}

The group comprised patients hospitalized in the Department of Anesthesiology and Intensive Care Medicine, Faculty of Medicine and Dentistry, Palacky University Olomouc and University Hospital Olomouc between 1 January 2009 and 31 December 2009 who developed signs of HAP. Also included were patients with positive findings in their tracheal secretion on admission to the department if transferred from another ward of the hospital. In intubated patients, secretion samples for microbiology culture tests were obtained by aspiration from the lower airway. Mortality was statistically analyzed by Fisher's exact test.

The clinical signs of pneumonia were defined as the presence of newly developed or progressive infiltrates on chest radiographs plus at least two other signs of respiratory tract infection: temperature $>38^{\circ} \mathrm{C}$, purulent sputum, leukocytosis $>10 \times 10^{3} / \mathrm{mm}^{3}$ or leukopenia $<4 \times 10^{3} /$ $\mathrm{mm}^{3}$, signs of inflammation on auscultation, cough and/ or respiratory insufficiency with oxygenation index $\mathrm{PaO}_{2} /$ $\mathrm{FiO}_{2} \leq 300 \mathrm{~mm} \mathrm{Hg}$.

Each sample was processed by semiquantitative method based on the four-quadrant streak technique using a calibrated loop. The etiologic agent was considered relevant if cultured in the tracheal secretion at a quantity of $>10^{5} \mathrm{CFU} / \mathrm{ml}^{1}$. Tracheal secretion samples were processed by traditional microbiological methods. The microorganisms were identified by the ENTEROtest 16 and STAPHYtest 16 (Lachema) biochemical identification tests, and the Phoenix (Becton, Dickinson and Company) or MALDI-TOF (Bruker Daltonics) automated systems. The yeasts were identified using the ID-32C (bioMérieux) commercial kit.

If an identical culture, determined as the etiologic agent, was repeatedly isolated from multiple samples obtained from the same patient, only the first isolated culture was included in the study.
Susceptibility to antibiotics was determined by the standard microdilution method according to the EUCAST criteria $^{6}$. Production of ESBL and AmpC broad-spectrum beta-lactamases was detected by modified DDST and $\mathrm{AmpC}$ tests ${ }^{7}$. Positive phenotype results were verified by polymerase chain reaction detecting the $b l a_{\text {CTX-M }}, b l a_{\mathrm{SHV}}$, and $b l a_{\text {TЕM }}$ genes $^{8,9}$.

All Staphylococcus aureus strains were tested for resistance to oxacillin by growth on the CHROMagar ${ }^{\mathrm{TM}}$ (Trios) selective diagnostic medium. Positive results were confirmed by detection of the mecA gene ${ }^{10}$.

Adequate initial therapy was defined as administration of at least one antibiotic (suitable for treating pneumonia) shown in vitro to be effective against the isolated causative agent.

Escherichia coli, Klebsiella pneumoniae, Pseudomonas aeruginosa and Burkholderia cepacia complex (BCC), were analyzed by comparing genomic DNA restriction fragments separated by pulsed-field gel electrophoresis (PFGE). Bacterial DNA was isolated according to Shi et al. and digested with restriction enzymes $\mathrm{Xba \textrm {I }}$ (Escherichia coli, Klebsiella pneumoniae, BCC) and SpeI (Pseudomonas aeruginosa) (Fermentas) ${ }^{11}$. PFGE was performed in $1.2 \%$ agarose gel. DNA fragments obtained by digestion with $X b a \mathrm{I}$ were separated for $24 \mathrm{~h}$ at $6 \mathrm{~V} \cdot \mathrm{cm}^{-1}$, pulse times of 2-35 s and $14{ }^{\circ} \mathrm{C}$. For reaction with SpeI, the following parameters were used: $23 \mathrm{~h}, 6 \mathrm{~V} . \mathrm{cm}^{-1}$, pulse times of 5-60 $\mathrm{s}$ and a temperature of $12{ }^{\circ} \mathrm{C}$. Subsequently, the gel was stained with ethidium bromide and photographed. The resulting restriction profiles were compared using the GelCompar II (Applied Maths) software.

\section{RESULTS}

From a total of 508 patients, 51 patients met the inclusion criteria (40 males and 11 females; male mean age 47.5 years, range $22-90$ years, median age 46 years; female

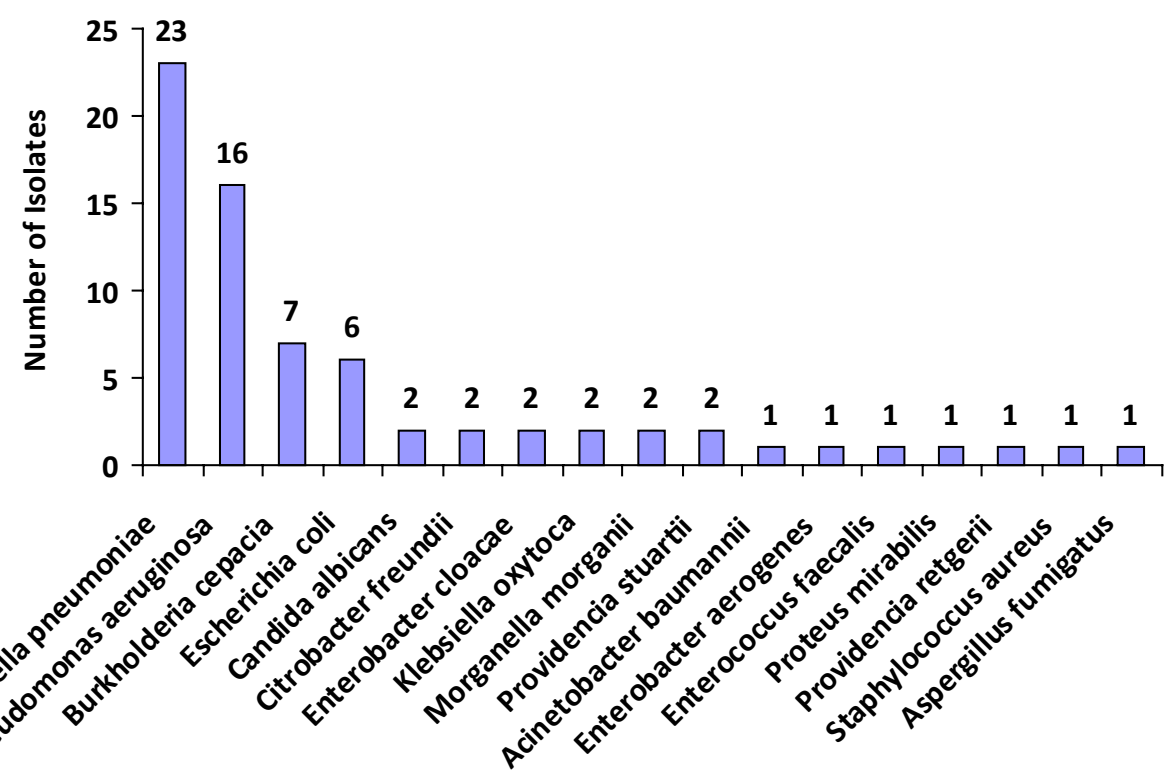

Fig. 1. Distribution of pathogens. 
Table 1. The numbers of pathogens and MDR strains by the type of pneumonia.

\begin{tabular}{|l|c|c|}
\hline Etiologic agent & $\begin{array}{c}\text { Early-onset } \\
\text { HAP (iso- } \\
\text { lates/MDR) }\end{array}$ & $\begin{array}{c}\text { Late-onset } \\
\text { HAP (iso- } \\
\text { lates/MDR) }\end{array}$ \\
\hline Aspergillus fumigatus & & 1 \\
\hline Acinetobacter baumannii & & $1 / 0$ \\
\hline Burkholderia cepacia & $1 / 1$ & $6 / 6$ \\
\hline Candida albicans & & 2 \\
\hline Citrobacter freundii & & $2 / 2$ \\
\hline Enterobacter aerogenes & & $1 / 0$ \\
\hline Enterobacter cloacae & & $2 / 1$ \\
\hline Enterococcus faecalis & & $1 / 1$ \\
\hline Escherichia coli & $1 / 0$ & $5 / 2$ \\
\hline Klebsiella oxytoca & & $2 / 1$ \\
\hline Klebsiella pneumoniae & $1 / 1$ & $22 / 11$ \\
\hline Morganella morganii & $1 / 0$ & $1 / 1$ \\
\hline Proteus mirabilis & & $1 / 0$ \\
\hline Providencia rettgeri & & $1 / 1$ \\
\hline Providencia stuartii & & $2 / 2$ \\
\hline Pseudomonas aeruginosa & & $16 / 9$ \\
\hline Staphylococcus aureus & & $1 / 0$ \\
\hline total strains/MDR: & $4 / 2$ & $67 / 37$ \\
\hline
\end{tabular}

Legend: MDR - multi-drug resistance, HAP - hospital-acquired pneumonia mean age 49.2 years, range 26-88 years, median age 45 years). Early-onset HAP was identified in 7 (14\%) patients ( 6 males, mean age 46.3 years, median age 45 years; 1 female, 88 years). Late-onset HAP was found in 44 (86\%) patients ( 34 males, mean age 51.8 years, median age 51 years; 10 females, mean age 46.6 years, median age 46 years).

The etiological agens of HAP were identified in 49 patients. In two patients, the etiology of HAP was not defined. In 14 patients (23\%), HAP was found to have polymicrobial etiology. The frequency of isolated pathogens was as follows: Klebsiella pneumoniae (32\%), Pseudomonas aeruginosa (22\%), BCC (10\%) and Escherichia coli (8\%) (Fig. 1).

Multidrug-resistant (MDR) strains were identified in $56 \%$ of Pseudomonas aeruginosa, 52\% of Klebsiella pneumoniae, 25\% of Escherichia coli and all the seven BCC (Table 1). Production of ESBL broad-spectrum betalactamases was found in 9 (39\%) Klebsiella pneumoniae isolates and in one (17\%) Escherichia coli. Constitutive production of AmpC broad-spectrum beta-lactamases was not detected in any of the tested species. The minimum inhibitory concentration values, required to inhibit the growth of $50 \%$ or $90 \%$ of organisms, and the percentage of resistance for individual antibiotic agents in strains detected in numbers greater than 10 are shown in Table 2.

During hospitalization, 18 patients died directly due to pneumonia, of which 16 were males and 2 were females (3 patients with early-onset and 15 patients with late-onset HAP). The most frequently isolated species associated with deaths were Klebsiella pneumoniae (10x, including

Table 2. $\mathrm{MIC}_{50}, \mathrm{MIC}_{90}$ and percentage of resistant isolates.

\begin{tabular}{|c|c|c|c|c|c|c|}
\hline \multirow{2}{*}{$\begin{array}{c}\text { Antibiotic } \\
\text { tested }\end{array}$} & \multicolumn{3}{|c|}{ Klebsiella pneumoniae } & \multicolumn{3}{|c|}{ Pseudomonas aeruginosa } \\
\hline & MIC50 & MIC90 & $\% \mathrm{R}$ & MIC50 & MIC90 & $\% \mathrm{R}$ \\
\hline AMI & 1 & 16 & 14 & 0.5 & 2 & 6 \\
\hline AMS & 32 & 64 & 90 & $=$ & $=$ & $=$ \\
\hline AZT & 8 & 64 & 53 & 8 & 16 & 39 \\
\hline CIP & 4 & 16 & 52 & 1 & 32 & 50 \\
\hline $\mathrm{COL}$ & 1 & 2 & 21 & $=$ & $=$ & $=$ \\
\hline COT & 16 & 128 & 68 & $=$ & $=$ & $=$ \\
\hline CPM & 2 & 16 & 54 & 8 & 16 & 55 \\
\hline CRX & 64 & 64 & 61 & $=$ & $=$ & $=$ \\
\hline CTX & 8 & 32 & 54 & $=$ & $=$ & $=$ \\
\hline CTZ & 8 & 32 & 54 & 2 & 16 & 33 \\
\hline GEN & 0.25 & 32 & 50 & 1 & 32 & 44 \\
\hline MER & 0.5 & 1 & 0 & 8 & 32 & 55 \\
\hline OFL & 4 & 16 & 54 & $=$ & $=$ & $=$ \\
\hline PIP & 128 & 256 & 80 & 16 & 64 & 61 \\
\hline PPT & 32 & 128 & 54 & 16 & 64 & 61 \\
\hline TIG & 0.25 & 0.5 & 0 & $=$ & $=$ & $=$ \\
\hline TOB & 32 & 32 & 54 & - & - & - \\
\hline
\end{tabular}

Legend: AMI-amikacin, AMS-ampicillin/sulbactam, AZT-aztreonam, CIP-ciprofloxacin, COL-colistin, COT-cotrimoxazole, CPM-cefepime, CRX-cefuroxime, CTX-cefotaxime, CTZ-ceftazidime, GEN-gentamicin, MER-meropenem, OFL-ofloxacin, PIP-piperacillin, PPT-piperacillin/ tazobactam, TIG-tigecycline, TOB-tobramycin 
Dice (Tol 1.5\%-1.5\%) (H>0.0\% S>0.0\%) [0.0\%-100.0\%] PFGE

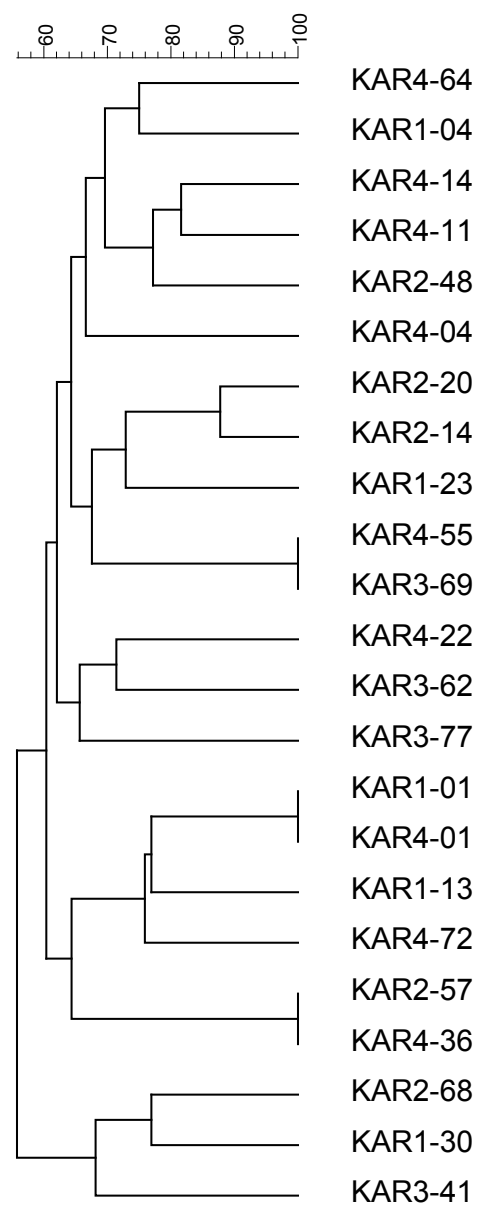

Legend: horizontal axis - similarity of bacterial isolates in \% vertical axis - isolate codes

Fig. 2. Dendrogram of Klebsiella pneumonia.

Dice (Tol 1.5\%-1.5\%) (H>0.0\% S>0.0\%) [0.0\%-100.0\%] PFGE

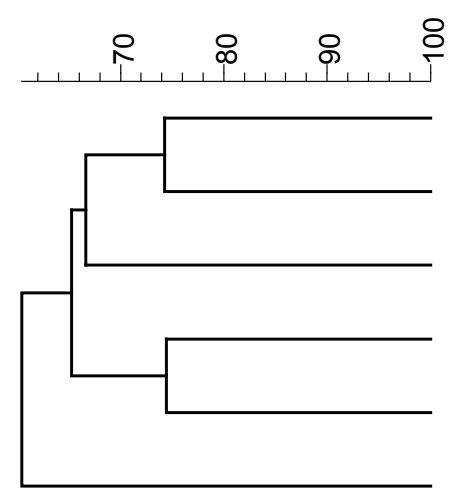

KAR1-24

KAR1-03

KAR4-23

KAR4-08

KAR4-47

KAR3-06

Legend: horizontal axis - similarity of bacterial isolates in \% vertical axis - isolate codes

Fig. 4. Dendrogram of Escherichia coli.
Dice (Tol $1.5 \%-1.5 \%)(H>0.0 \%$ S $>0.0 \%)[0.0 \%-100.0 \%]$
PF PFGE

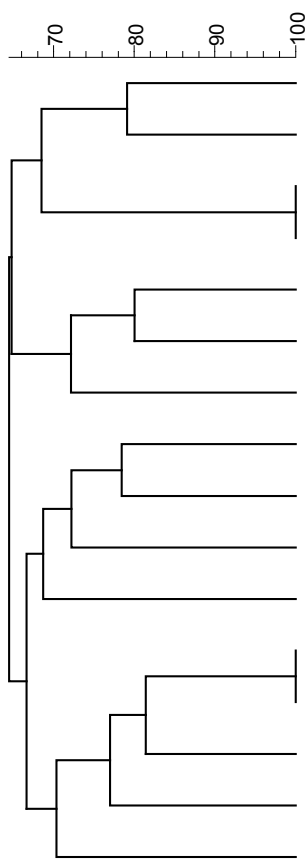

KAR4-15

KAR4-37

KAR3-72

KAR4-03

KAR1-77

KAR2-24

KAR1-28

KAR1-20

KAR2-30

KAR1-34

KAR2-42

KAR3-71

KAR3-52

KAR2-32

KAR3-14

KAR1-07

Legend: horizontal axis - similarity of bacterial isolates in \% vertical axis - isolate codes

Fig. 3. Dendrogram of Pseudomonas aeruginosa.

Dice (Tol 1.5\%-1.5\%) (H>0.0\% S>0.0\%) [0.0\%-100.0\%] PFGE

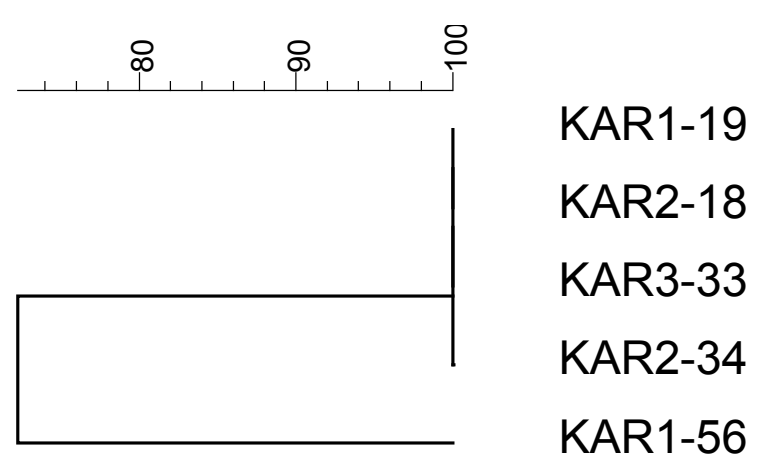

Legend: horizontal axis - similarity of bacterial isolates in \% vertical axis - isolate codes

Fig. 5. Dendrogram of Burkholderia cepacia complex. 
5 MDR strains), Pseudomonas aeruginosa (7x, 4 MDR) and BCC (6x, all MDR). In patients whose death was directly associated with late-onset HAP, the most frequent species cultured from tracheal secretions were Klebsiella pneumoniae (29\%), Pseudomonas aeruginosa (23\%) and BCC (16\%).

Comparison of data on bacterial resistance to antimicrobial agents with the administered initial antibiotic therapy and deaths directly related to HAP suggested that with adequately selected initial treatment, 21 patients survived but 9 died. On the other hand, with inadequate initial antibiotic therapy, 9 patients died and 12 survived. Thus, the mortality rates were $30 \%$ and $43 \%$ for adequate and inadequate therapy, respectively.

From a total of 52 (Escherichia coli, Klebsiella pneumoniae, Pseudomonas aeruginos and BCC) strains, 50 of them were compared by PFGE. In 2 BCC isolates, genomic DNA of quality sufficient for restriction analysis could not been repeatedly isolated.

Out of 23 Klebsiella pneumoniae isolates, an identical restriction profile was found in 3 pairs obtained from 6 different patients (Fig. 2). In all cases, the identical strains were collected at intervals longer than 4 months. Among 16 isolates of Pseudomonas aeruginosa, 2 pairs of identical strains isolated in 4 different patients were detected, with the intervals between collections being shorter than 1 month (Fig. 3). In case of Escherichia coli, all compared strains were genetically different (Fig. 4). In the BCC group, however, 4 out of 5 tested isolates collected between 19 January and 14 March 2009 from different patients were identical (Fig. 5).

\section{DISCUSSION}

Given the incidence of 51 cases out of a total of 508 patients hospitalized in the Department of Anesthesiology and Intensive Care Medicine in 2009, the prevalence (10\%) was at the lower limit of the commonly reported range $(9-24 \%)\left(\mathrm{ref}^{3,12}\right)$. In agreement with the literature, most cases $(86 \%)$ were late-onset HAP.

Bacterial pathogens associated with the majority of deaths were strains of Klebsiella pneumoniae, Pseudomonas aeruginosa, BCC and Escherichia coli, more than 50\% of which were multiresistant. In late-onset HAP, MDR strains accounted for as many as $58 \%$ of strains. In earlyonset HAP, the proportion of MDR strains could not been reliably assessed due to the small number of cases.

The mortality rates in patients receiving adequate initial antibiotic therapy were $13 \%$ lower than in those treated with antibiotics to which isolated bacterial pathogens were later found to be resistant. According to Fiser's two-sided exact test, the resulting difference in mortality rates between the two studied groups is not significant $(P=0.385)$, because of small set of data. Given the fact that despite adequate initial antibiotic therapy, the mortality rates were relatively high ( 9 out of 30 dead patients) and mortality is also likely to be influenced by the time of initiation of antibiotic therapy.
In case of the mortality rates in patients with adequate therapy, our results $(30 \%)$ are in agreement with those by Luna et al. who reported 38\% $\left(\mathrm{ref}^{4}\right)$. The same authors also found $91 \%$ mortality associated with inadequate therapy, a much higher rate than our results (43\%). The difference, however, might be explained by a different epidemiological situation at bacterial resistance levels.

Although in Klebsiella pneumoniae and Pseudomonas aeruginosa, five pairs of identical strains were noted in 10 patients, most isolated strains were unique and HAP may be characterized as endogenous. Despite the high proportion of MDR strains, clonal spread was not confirmed, with the exception of BCC. Thus, secondary colonization of patient's microflora during hospitalization is likely.

Four identical BCC cultures were identified as Burkholderia multivorans by the MALDI-TOF system. Since those were isolated within a very short period of time, they are likely to constitute a new epidemic strain of Burkholderia multivorans, similar to Burkholderia cenocepacia in patients with cystic fibrosis ${ }^{13}$.

\section{CONCLUSION}

Adequate empirically selected therapy must be based on the current knowledge of epidemiological situation in the region, but especially in the hospital and the particular department. The study confirmed higher mortality rates in the group of patients with inadequately selected initial antibiotic therapy. Most strains isolated in the study were unique. Thus, HAP may be characterized mostly as endogenous, with the source being the patient himself or herself. The study results suggest high-quality barrier nursing care. However, measures to prevent contamination of the airways by the patient's own flora must be considered. Four identical BCC cultures were identified as Burkholderia multivorans and clonal spread of the strains may be assumed.

\section{ACKNOWLEDGEMENTS}

Supported by the grant projects MSM6198959205 and LF_2011_002.

\section{REFERENCES}

1. Joseph NM, Sistla S, Dutta TK, Badhe AS, Parija SC. Ventilatorassociated pneumonia: A review. Eur J Intern Med 2010;21:360-8.

2. Woodhead M, Blasi F, Ewig S, Huchon G, Leven M, Ortquist A, Schaberg T, Torres A, Van der Heijden G, Verheij TJM. ERS task force in collaboration with ESCMID. Guidelines for management of adult lower respiratory tract infections. Eur Respir J 2005;26:1138-80

3. Thomas M File. Epidemiology, pathogenesis, microbiology, and diagnosis of hospital-acquired, ventilator-associated, and healthcareassociated pneumonia in adults. UpToDate (2011) [cited 27 May 2011]. Available from: http://www.uptodate.com/home/index.html

4. Luna CM, Vujacich P, Niederman MS, Vay C, Gherardi C, Matera J, Jolly EC. Impact of BAT data on the therapy and outcome of ventilator-associated pneumonia. Chest 1997;111:676-85. 
5. Rello J, Torres A, Ricart M, Valles J, Gonzales J, Artigas A, Rodriques-Roisin R. Ventilator-associated pneumonia ba Staphylococcus aureus. Comparison of methicillin-resistant and methicillin-sensitive episodes. Am J Respir Crit Care Med 1994; 150:1545-9.

6. European Committee on Antimicrobial Susceptibility Testing Breakpoint tables for interpretation of MICs and zone diameters. Version 1.3, 5 January 2011 [cited 27 May 2011]. Available from: http://www.eucast.org/clinical breakpoints

7. Htoutou Sedlakova M, Hanulik V, Chroma M, Hricova K, Kolar M, Latal T, Schaumann R, Rodloff AC. Phenotypic detection of broad-spectrum beta-lactamases in microbiological practice. Med Sci Monit 2011;17:BR147-52.

8. Pérez-Pérez FJ, Hanson ND. Detection of plasmid-mediated AmpC $\beta$-lactamase genes in clinical isolates by using multiplex PCR. J Clin Microbiol 2002;40:2153-62.

9. Pagani L, Dell'Amico E, Migliavacca R, D Andrea MM, Giacobone E, Amicosante G, Romero E, Rossolini GM. Multiple
CTX-M-type extended-spectrum -lactamases in nosocomial isolates of Enterobacteriaceae from a hospital in northern Italy. J Clin Microbiol 2003;41:4264-9.

10. Oliveira DC, de Lencastre H. Multiplex PCR strategy for rapid identification of structural types and variants of the mec element in methicillin-resistant Staphylococcus aureus. Antimicrob Agents Chemother 2002;46:2155-61.

11. Shi ZY, Liu PYF, Lau YJ, Lin YH, Hu BS. Epidemiological typing of isolates from an outbreak of infection with multidrug-resistant Enterobacter cloacae by repetitive extragenic palindromic unit b1primed PCR and pulsed-field gel electrophoresis. J Clin Microbiol 1996;34:2784-90.

12. Vincent JL. Nosocomial pneumonia. Indian J Crit Care Med. 2001;5:148-56.

13. Drevinek P, Vosahlikova S, Cinek O, et al. Widespread clone of Burkholderia cenocepacia in cystic fibrosis patients in the Czech Republic. J Med Microbiol 2005;54:655-9. 